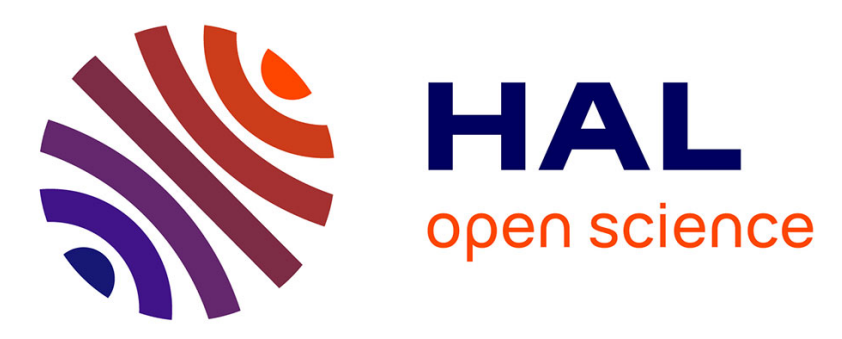

\title{
Modulating Portal Hemodynamics With Vascular Ring Allows Efficient Regeneration After Partial Hepatectomy in a Porcine Model.
}

Petru O. Bucur, Mohamed Bekheit, Chloe Audebert, Amnah Othman, Seddik Hammad, Mylène Sebagh, Marc Antoine Allard, Benoît Decante, Adrian Friebel, Dirk Drasdo, et al.

\section{To cite this version:}

Petru O. Bucur, Mohamed Bekheit, Chloe Audebert, Amnah Othman, Seddik Hammad, et al.. Modulating Portal Hemodynamics With Vascular Ring Allows Efficient Regeneration After Partial Hepatectomy in a Porcine Model.. Annals of Surgery, 2017, 10.1097/SLA.0000000000002146 . hal-01494844

\author{
HAL Id: hal-01494844 \\ https://hal.science/hal-01494844
}

Submitted on 24 Mar 2017

HAL is a multi-disciplinary open access archive for the deposit and dissemination of scientific research documents, whether they are published or not. The documents may come from teaching and research institutions in France or abroad, or from public or private research centers.
L'archive ouverte pluridisciplinaire HAL, est destinée au dépôt et à la diffusion de documents scientifiques de niveau recherche, publiés ou non, émanant des établissements d'enseignement et de recherche français ou étrangers, des laboratoires publics ou privés. 


\section{Modulating portal hemodynamics with vascular ring allows efficient regeneration after partial hepatectomy in a porcine model}

Petru O. Bucur 1,2, Mohamed Bekheit 1,2, Chloé Audebert 3,4, Amnah Othman 5, Seddik Hammad 5,6, Mylene Sebagh 7, Marc-Antoine Allard 1, Benoît Decante 8, Adrian Friebel 9, Dirk Drasdo 3,9, Elodie Miquelestorena-Standley 10, Jan G. Hengstler 6, Irene Vignon-Clementel 3,4, Eric Vibert 1,2.

\section{Authors`affiliations}

1. AP-HP, Hôpital Paul Brousse, Centre Hépato-Biliaire, Villejuif, France

2. Inserm Unité 1193, Villejuif, France

3. INRIA Paris-Rocquencourt, France

4. Sorbonne Universités, UPMC Univ. Paris 6, Laboratoire Jacques-Louis Lions, Paris, France

5. Leibniz Research Centre for Working Environment and Human Factors (IFADO), TU Dortmund University, Dortmund, Germany

6. Department of Forensic Medicine and Veterinary Toxicology, Faculty of Veterinary Medicine, South Valley University, Qena, Egypt

7. AP-HP, Hôpital Kremlin-Bicètre, Pathology Department, Kremlin-Bicètre, France

8. Centre Chirurgicale Marie-Lannelongue, Experimental Surgery Unit, Le Plessis Robinson, France

9. Interdisciplinary centre for Bioinformatics (IZBI), University of Leipzig, Leipzig, Germany

10. CHRU Tours, Laboratoire d'anatomie et cytologie patholoiques, Tours, France 


\section{Corresponding author:}

EricVibert, MD, PhD

12 Avenue Paul VaillantCouturier

94804 Villejuif Cedex

Tel: 0033145593000

Fax: 0033145593857

E-Mail: eric.vibert@pbr.aphp.fr

Key words: Post-hepatectomy failure, Small-for-size, liver resection, portal flow modulation, vascular ring, liver regeneration.

\section{Conflict of interest:}

Eric Vibert and Petru Bucur were consultants for MID, Dardilly, France. The rest of authors declare no conflict of interest.

Source of funding: this study was funded mainly by the "Agence de la Biomedecine" through its program of Research (AOR 2009) and Société Francophone de la Transplantation (Bourse IGL 2009). Eric Vibert, Irene Vignon-Clementel, Dirk Drasdo, Petru O. Bucur, Mohamed Bekheit, Chloé Audebert, and Jan Hengestler acknowledge funding by project ANR-13-TECS-0006 (IFlow), Adrian Friebel by Virtual Liver Network (German Bundesministerium für Bildung und Forschung [BMBF]).

Authors`contribution:

Study concept and design: Eric Vibert, Irene Vignon-Clementel 
Acquisition of data: Mohamed Bekheit, Petru Bucur, Marc-Antoine Allard, Chloé Audebert, Benoît Decante

Analysis and interpretation of data: Mohamed Bekheit, Chloé Audebert, Petru

Bucur, Amnah Othman, Seddik Hammad, Mylene Sebagh, Adrian Friebel, Dirk Drasdo,

Eric Vibert, Irene Vignon-Clementel, Elodie Miquelestorena-Standley

Drafting of the manuscript: Mohamed Bekheit, Petru Bucur, Chloé Audebert

Final approval: Eric Vibert, Irene Vignon-Clementel, Jan G. Hengstler, D Drasdo

Disclosure: The authors disclose that the percutaneously adjustable vascular ring described in the manuscript is still investigational.

Key words:

Post-hepatectomy failure, Small-for-size, liver resection, portal flow modulation, vascular ring, liver regeneration. 


\section{Introduction}

The liver function is dependent on the integrity of the micro-architecture that permits optimum exchange of metabolites between blood and hepatocytes (1). After resection, the portal flow through the sinusoidal network increases leading to stimulation of sinusoidal endothelial cells (2) and initiation of liver regeneration (3). Major hepatectomy or transplantation of small liver are, on the other hand, associated with disequilibrium between the portal flow rate, which is excessively increased at the sinusoidal level, and the liver volume, leading to "barotrauma" $(4,5)$.

The volume and the quality of the future liver are important determinants for this disequilibrium $(6,7)$. Thresholds for portal flow rate per $100 \mathrm{~g}$ of liver (8) and portal pressure (9) were identified above which the risk of post-operative liver failure is high (10). This phenomenon is partially interplayed by the important reduction in the arterial flow "de-arterialization" of the remnant liver as a consequence of the excess in portal flow rate through the sinusoidal network (11).

Healthy remnant liver volume superior to $20 \%$ of the theoretical total liver volume (12) and/or superior to $0.5 \%$ of the body weight is considered mandatory after major hepatectomy $(13,14)$ to keep a balance between volume and flow to avoid post-operative liver failure. If more resection is anticipated, which is the case in many liver malignancies, preoperative portal vein occlusion, by embolization or ligation, might be necessary to induce regeneration of the future liver (15). However, this approach may increase the risk of cancer progression $(16,17)$. 
Portal flow modulation was initially applied in living donor liver transplantation (18).

Partial portal flow diversion (19), splenic artery ligation (20) or splenectomy were

proposed to reduce the incidence of post-operative liver failure $(21,22)$. These techniques do not allow precise control of the portal flow rate and might have adverse effects. For instance, excessive diversion of flow might have an equally deleterious effect on liver regeneration (22), to which it is essential (3). Therefore, it would be helpful to use a modulation technique with flexible and reversible control over the portal hemodynamics to tailor it to the planned remnant volume.

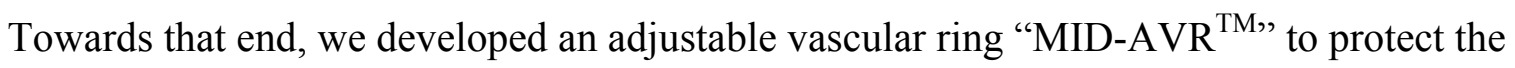
hepatic microarchitecture from the initial harmful barotrauma._The efficacy of the ring was assessed in terms of survival, liver function tests, liver regeneration, and changes in the micro-architecture.

\section{Materials and Methods:}

Ethical approval: The study was approved by the regional committee of ethics of animal research, and by the French Government authorities, complying with the European Union Directive $N^{\circ}$ 2010/63/EU.

Animals: All animals received humane care according to the criteria outlined in the "Guide for the Care and Use of Laboratory Animals" prepared by the National Academy of Sciences and published by the National Institutes of Health (23). Seventeen large white female pigs, which underwent $75 \%$ liver resection, were randomised in blocks into two groups. The control (no MID-AVR ${ }^{\mathrm{TM}}$ ) group included 9-animals and the ring group $\left(\mathrm{MID}-\mathrm{AVR}^{\mathrm{TM}}\right.$ ) included 8-animals in which the vascular ring was positioned around the 
portal vein. The average age of the included animals was 3 months \pm 9 days and their mean weight was $32.9 \pm 5.3 \mathrm{~kg}$.

Study setting: Surgeries were performed at the experimental animal surgical unit at the Marie Lannelongue Center, Le Plessis Robinson, France.

Preoperative preparation: Animals were left fasting the night before surgery. On the day of surgery, animals were given in their individualized cages $30 \mathrm{mg} / \mathrm{kg}$ ketamine (Ketamin, Panpharma) and $0.03 \mathrm{mg} / \mathrm{kg}$ acepromazine (Calmivet, Vetoquinol, France).

Anaesthesia: All surgeries were performed under general anaesthesia. Each pig received $100 \mathrm{mg}$ of xylazine 2\% (Rompun, Bayer Healthcare) with $750 \mathrm{mg}$ ketamine for anaesthesia induction followed by tracheal intubation (6-7 $\mathrm{mm}$ in size, Portex, France). Subsequently, inhalational anaesthesia was started using a $60 \% \mathrm{FiO} 2$ with $2 \%$ isoflurane (Isoflurane, Belamont, France) in assisted ventilation.

Pancuroniumbromide (Pavulon, Schering-Plough), at a rate of $0.3 \mathrm{mg} / \mathrm{kg} / \mathrm{h}$ and fentanyl (Fentanyl Janssen $100 \mu \mathrm{g} / 2 \mathrm{ml}$ ), at a rate of $5 \mu \mathrm{g} / \mathrm{kg} / \mathrm{h}$ were continuously perfused intravenously. Crystalloid fluids were given at a rate of $2 \mathrm{ml} / \mathrm{kg} / \mathrm{h}$-fasting in addition to $500-1000 \mathrm{ml}$, which was increased as required. At the end of surgery, the wound was infiltrated with ropivacaïne 150mg (Naropeine, AstraZeneca, $7.5 \mathrm{mg} / \mathrm{ml}$ ).

During surgery animals were covered with heat blankets and gastric aspiration through an oro-gastric tube was attempted if gastric distension was observed.

Cefotaxime 1g (Cefotaxime, Mylan) and gentamicine 80mg (Gentalline, ScheringPlough) were given intramuscularly once a day for 5-days. In addition, animals received 
Pantoprazole 40mg/i.v (Inipomp, Nycomed) and enoxaparine $0.2 \mathrm{ml} / \mathrm{S} . \mathrm{C}$ (Lovenox, Sanofi Aventis) and 0.5mg/kg/b.i.d Nalbuphine (Nalbuphine Serb, 20mg/2ml).

The same preoperative measures were repeated at the time of sacrifice on the 7 th postoperative day. Blood samples were collected before and after liver resection as well as on the $3^{\text {rd }}, 5^{\text {th }}$, and the $7^{\text {th }}$ postoperative days.

\section{Surgical procedure:}

Hemodynamic measurements: Median cervical incision and cannulation of the right internal jugular vein with an $8 \mathrm{Fr}$ and the right carotid artery with a $5 \mathrm{Fr}$ Desivalve (Vygon, Ecouen, France) vascular cannula were performed.

Sternotomy was performed to place the TranSonic $20 \mathrm{~mm}$ (20 PAX, TranSonic, Ithaca, NY, USA) transit time echo probe around the origin of the ascending aorta for measuring the cardiac output. Subsequently, a midline abdominal incision was performed. Upon dissecting the hepatic hilum, two other flow meter probes, $14 \mathrm{~mm}$ and $4 \mathrm{~mm}$, were positioned around the portal vein and the hepatic artery; respectively. Portal and venacava pressures were measured by direct puncture with a $24 \mathrm{G}$ needle connected to a builtin electronic transducer in the anaesthetic monitor.

Flow per unit mass was calculated as the recorded flow rate in the main portal vein divided by the liver weight multiplied by 100 ( $\mathrm{ml} /$ minute/100 gram of liver tissue), and the whole liver weight was estimated based on the fact that the left lateral, the left medial and the right medial lobes of the pig liver constitute around $75 \%$ of the whole liver weight (24). The whole liver weight is calculated as the resected liver weight *100/75. 
MID-AVR ${ }^{\text {TM }}$ positioning: The ring is silicone made (figure $1 \mathrm{a}, \mathrm{b}$ ); connected to a regulating valve via long tube. The two lips of the ring opening were fixed together with a fine non absorbable (polypropylene 8/0) suture that is readily broken upon over inflation of the balloon (figure 1c) (supplemental video 1 demonstrates the inflation process). The ring was placed around the portal vein (figure 1d) in the designated group and calibrated before starting liver resection.

Once the ring was placed, the balloon was progressively inflated with sterile saline solution with $0.1 \mathrm{ml}$ steps. At each step the flow rate in the portal vein as well as the pressure below and above the ring were measured during 4-5 minutes to ensure the stability of the effect. The target portal flow rate in the MID-AVR ${ }^{\mathrm{TM}}$ group was $50 \%$ of its initial value, to limit the increase in the portal flow per the remnant liver mass after a $75 \%$ liver resection to around twofold (25).

At the end of surgery, the valve was fixed subcutaneously on the xiphoid process with non-absorbable sutures for percutaneous control and extraction.

Liver resection: Resection of the left lateral, left medial and the right medial hepatic lobes was done leaving in place the right lateral and the caudate lobes.

At the end of the procedure, a central venous catheter was placed in the internal jugular vein for postoperative fluid administration and blood samples withdrawal. Biopsies from the remnant liver lobe were taken before and one hour after liver resection. Pleuromediastinal suction drain was placed at the end of the procedure and removed on the first postoperative day. 
Fifteen-minute indocyanine green retention\% (ICG-R15): After resection, ICG-R15 was measured in serum from arterial samples taken before injection of $0.5 \mathrm{mg} / \mathrm{kg}$ ICG (Infracyanine, SERB, France) and 2, 4, 8 and 15 minutes after the injection.

Day-3 postoperative: An ultrasound guided liver biopsy using an 18 Fr needle was taken. In the MID-AVR ${ }^{\mathrm{TM}}$ group, removal of the ring was performed by reopening the midline incision in the first three animals, in order to ensure that the ring was completely open and that the anchoring sutures ruptured. Subsequently, it was removed through a small percutaneous incision over the valve.

Sacrifice: On the $7^{\text {th }}$ postoperative day, animals were sacrificed following a similar protocol to that at the day of surgery. The remnant liver was weighted after euthanasia.

\section{Histological analysis:}

Pathological scores were given to specimens in standard hematoxylin-eosin and Masson trichrome stains based on criteria adapted from Demetris et al (26). Five criteria were formulated: $\mathrm{A}=$ cellular proliferation in clusters, $\mathrm{B}=$ sinusoidal dilatation, $\mathrm{C}=$ ballooning, $\mathrm{D}=$ ductular proliferation and $\mathrm{E}=$ inflammatory necrosis. Each item is graded from $0=$ no change, $1=$ mild, $2=$ moderate, to $3=$ severe, in samples taken from deep and superficial liver tissue for a total score between 0 and 30 for the liver at each time point. An experimented pathologist (MS) assigned blindly all the values that were used for the scores. A second pathologist (EMS) familiar with pig liver pathology reviewed independently all samples and established another set of values.

Proliferation index and 3D morphometric quantification of the bile canaliculi: 
Five-micron sections of formalin-fixed paraffin-embedded liver tissues were processed according to previously published protocols (27). Likewise, in order to threedimensionally reconstruct and analyse the bile canalicular network, $100 \mu \mathrm{m}$ liver slices were immunostained. Subsequently, Z-stacks ( $\mathrm{n}=6-9$ per group) were captured by a 60 fold objective using a confocal laser scanning microscope (FV1000, Olympus, Germany). Image preprocessing was carried out by Autoquant-X3 Version X3.03 64 Bit Edition (Bitplane). Image segmentation for quantification of bile canaliculi was achieved by TiQuant (www.msysbio.com/tiquant) using a voxel size of $0.207 \mu \mathrm{m} \times 0.207 \mu \mathrm{m} \times 0.54$ $\mu \mathrm{m}$ was used (28) (Supplemental movies 2-4). For morphometric quantification, bile canaliculi were classified into branches and nodes (footnote supplementary table 3).

\section{Statistical analysis:}

After normality testing, summary of data were represented in mean \pm standard deviation, median and range or percentages according to variable type. Odds ratio was reported to compare mortality between both groups.

Man-Whitney-U test and Chi-square tests were used to assess the difference between the control and the ring groups for non-parametric variables, while $t$-test was calculated for parametric variables. ANOVA with repeated measures and Friedman tests were used to compare the evolution of parameters for parametric and non-parametric data respectively. Significance threshold was set at a p-value of 0.05. MedCalc Statistical Software version 14.8.1 (MedCalc Software bvba, Ostend, Belgium), was used for the analysis.

\section{Results:}


The experimental design was set to study the effect of MID-AVR ${ }^{\mathrm{TM}}$ application, compared to no-application, on liver regeneration after $75 \%$ resection in two groups of large white pigs. Hemodynamic, laboratory, and histopathological parameters were analysed at different time points as indicated in the methods. Both groups had similar baseline parameters (supplementary table 1).

Ring safety: Application of the ring was easy and its removal was safe in all animals. In the first three animals, removal under visual control demonstrated the efficiency of the over inflation to open the ring. Percutaneous removal in the subsequent animals was similarly safe and successful. Patency of the portal vein was confirmed in the surviving animals by ultrasound at day- 3 and by direct visualization at sacrifice. The main difficulty was closing the ring with such a fine suture. But this was necessary for the opening of the ring by over inflation.

Survival: Six pigs (75\%) in the MID-AVR ${ }^{\mathrm{TM}}$ group and 5 pigs (55.6\%) in the control group survived till day-7 $(\mathrm{p}=0.62)$. However, when portal flow per unit liver mass at the end of surgery was within the range of 2.2-4 folds its baseline, only one animal died prematurely (10\%). On the other hand, 5 animals $(71 \%)$ died when these values were outside the range $(\mathrm{p}=0.017)$ (odds ratio $=22, \mathrm{p}=0.02)$ (figure 2$)$.

Autopsy was performed for all premature deaths, and it was negative for macroscopic explanation for the mortality. None of the pigs showed portal vein thrombosis.

Hemodynamic measurements: Portal flow rate after the ring placement, but before hepatectomy, was $80 \mathrm{~mL} / \mathrm{min} / \mathrm{g} * 100 \mathrm{~g}, 45 \%$ lower than its baseline value $(142 \mathrm{~mL} / \mathrm{min} / \mathrm{g} * 100 \mathrm{~g})$. The hemodynamics measured at the end of hepatectomy are 
presented in table 1. In the ring group, the porto-caval pressure gradient was significantly lower than in the control group $(\mathrm{p}<0.01)$ (figure 3a). In a stepwise multiple regression analysis, which included the changes in the parameters of systemic circulation as well as the presence or absence of the ring, only the last variable was a significant predictor of the change of porto-caval pressure gradient $(\mathrm{p}=0.001)$ whereas the systemic hemodynamic changes were not significant predictors. There was no difference in the pressure gradient at the post-operative day-7 between the two groups (i.e. 4 days after ring removal).

Changes in liver weight: The estimated residual liver weight was not significantly different between the control and the ring groups $(155 \pm 32$ gram and $151 \pm 25$ gram, respectively, $\mathrm{p}=1$ ). The residual liver weight increased significantly at day- $7, \mathrm{p}<0.0001$ (507 \pm 77 grams and $478 \pm 37$ grams in the ring and the control groups respectively) (figure 3b). There was no significant difference in the liver weight between both groups at the day of sacrifice, but the estimated gain was slightly higher in the ring group with no statistically significant difference (mean difference $=353 \pm 68 \mathrm{ml}$ versus $323 \pm 36 \mathrm{ml}$, respectively).

Laboratory results: The MID-AVR ${ }^{\mathrm{TM}}$ was a significant influential factor on the bilirubin level in repeated measures ANOVA ( $\mathrm{p}=0.033$ ). Postoperatively, serum bilirubin level was lower in the MID-AVR ${ }^{\mathrm{TM}}$ group at day 5, than in the control group (3.8 vs 6.6 $\mu \mathrm{mol} / \mathrm{L}, \mathrm{p}=0.007$ ) (figure $4 \mathrm{a}$ ). We observed slightly higher prothrombin activity values, 
after hepatectomy (figure $4 \mathrm{~b}$ ) in the ring group with no significant difference ( $\mathrm{p}=\mathrm{ns})$. For the rest of the parameters, no differences were observed. Table 1 summarizes the main findings after resection and at sacrifice.

Indocyanine green retention\%: [ICG-R15] was not significantly different between groups. However, the average retention\% was slightly lower at day-7 in the ring group compared to the control group (figure 4c).

Histopathological results: The overall changes in the total histopathological scores were significantly different between both groups $(\mathrm{p}=0.004)$. These differences were mainly in specimens after resection and at day 3 ( $\mathrm{p}<0.05$ in both time points). These differences remained significant when we used the mean of values from our two pathologists. (supplemental table 2 and 3) Sinusoidal dilatation was significantly lower in the ring group compared to the control at the end of surgery $(\mathrm{p}<0.01)$ (figure $5 \mathrm{a}-\mathrm{f})$. The Ki67 positive cells increased by $14.3 \%(95 \% \mathrm{CI}=10.8-17.9 \%)$ at post-operative day-3 compared to before surgery and decreased again at day- 7 with a difference in the activity of $1.5 \%(95 \% \mathrm{CI}=-2.8$ to $-0.2 \%)$ compared to baseline. Between the two groups, the percentage of Ki67 positive cells was higher at day-3 $(\mathrm{p}=0.043)$ in the ring group. The means of mitotic figures were not statistically different between groups, even if there were slightly less mitoses per field in the ring group at day $3(1.14$ vs $2, p=0.12)$ and more at day $7(3.17$ vs $1.8, \mathrm{p}=0.5)$.

In 3D morphometric quantification of the biliary structures, the number of intersection branches and the number of intersections nodes were significantly lower in the control group (but not in the ring group) at day-7 compared to the values before resection (mean 
difference $=162$ branch, $95 \% \mathrm{CI}=44-279$ intersection branch and mean difference $=130$ node, $95 \% \mathrm{CI}=13-248$ intersection node, [supplemental table 4]). The cumulative length of bile canaliculi was significantly lower at day- 7 than in preoperative specimen in both groups ( $\mathrm{p}=0.02)$ (figure $6 \mathrm{c}, \mathrm{d})$. (supplemental movies 2-4).

\section{Discussion:}

This present study presents the effects of a novel, adjustable, and less invasive technique for portal hemodynamics modulation on liver regeneration after $75 \%$ hepatectomy in a porcine model.

The application of the ring was safe and associated with better hepatic function represented in the lower bilirubin levels during the 5th post-operative day as well as in the less histological derangement and changes in the temporal proliferation pattern indicated by a higher Ki67 index at day 3 while the liver mass at day 7 in both groups did not differ. Application of the ring was not easy, especially the closure with the fine suture. Its utilisation seemed safe in pigs but liver position is different in humans and changes to adapt its position and avoid conflicts with other hepatic pedicle elements might be necessary.

The proliferative activity seemed to occur later in the ring group (more Ki67 activity and slightly less mitoses at day 3 and slightly more mitoses at day 7). Together with the higher pathological scores these results indicate that the control group had a temporally different cellular proliferation and a deranged micro-architecture that could be attributed to the effect of barotrauma. Sinusoidal barotrauma is thought to be a leading mechanism 
of post-operative liver failure $(5,20)$. In our study, each one of the animals in the ring group with their portal flow within this range of 2.2-4 folds survived.

In this study, the majority of animals at the end of surgery in both groups had an estimated portal flow per unit mass within the target range. However, the ring group showed lower portocaval pressure gradient, which indicates that the intra-sinusoidal pressure was lower in ring group. Indeed, the flow in ring group after ring positioning does not reflect the intra-parecnhymal resistance or pressure since it reflects the resistance imposed by the ring positioning, unlike the case in the control group. The parenchyma in the ring group continues to receive a lower flow compared to the control group, which leads to lower intra-sinusoidal pressure, smaller portocaval pressure gradient and lesser architectural damage. Therefore, we conclude that the ring application helped in protecting the hepatic microarchitecture in the initial phase after resection.

The higher porto-caval pressure gradient, which is a manifestation of increased microvascular resistance (29), explains the more evident changes in the microachitecture in the control group despite that there was no difference in the calculated portal flow per unit mass. Furthermore, the higher bilirubin and ICG-R15 level, which are typical manifestations of portal hyperperfusion (30), could be attributed to the higher intrasinusoidal pressure in this group. Alone; the high bilirubin level was reported to be a sufficient laboratory feature for the diagnosis of post-hepatectomy liver failure (31). The aforementioned changes imply that the better hepatic function in the ring group was attributed to the maintained microarchitectural integrity in this group. 
The relation between the portal pressure and the portal flow rate is not strictly linear owing to the interplay of the hepatic sinusoidal capacitance, which means that it would usually require more than doubling of the portal flow through the sinusoidal network for the portal pressure to slightly increase (32). Given the capacitance of the liver as a reservoir for blood (33), the absence of increased porto-caval pressure gradient in the ring group reflects the lower intra-sinusoidal pressure in spite of the relatively higher portal flow calculated from flow rate proximal to the ring in this group.

An increase in portal flow per liver mass is thought to be necessary for the stimulation of liver regeneration (22) and is associated with increased cellular proliferation through proliferative gene expression and apoptotic gene down regulation (34). On the contrary if there is an excessive increase in portal flow, liver functions are deranged (35) and suppression of liver regeneration paradoxically dominates, leading to liver failure (36).

The liver weight gain was lower in the control group than in the ring group. Furthermore, in the 3D quantification of biliary structures, all morphometric parameters had lower values in both groups compared to specimens taken before resection, indicating a higher hepatocyte density, as supported by the interpretation of the pattern of Ki67 index. . These parameters were higher in the ring group than in the control group implying that the regeneration in the ring group was more balanced and organised, which might explain the better hepatic function in this group.

Hepatic inflow modulation is becoming an increasingly accepted strategy for the reduction of the initial damage caused by the small for flow syndrome $(37,38)$. It targets 
the early protection of the architecture from the barotrauma inflicted by high portal flow during the inductive angiogenesis phase (2).

Several techniques have been proposed for modulation of the portal inflow. Diversion of the portal flow through a partial porto-caval shunt would require a second intervention for the closure of the shunt (39). Splenectomy offered more benefit compared to the portal flow diversion in terms of liver regeneration (38). This could be attributed to the negative effect of the excessive diversion on regeneration (40). Splenic artery embolization, as an alternative, exposes the spleen to the risk of infarction (41). Those alternatives do not have the dynamic and minimal invasive advantages offered by the reversible modulation with an adjustable vascular ring, which could be tailored to the extent of the planned liver resection.

In this study, the application of the ring prevented the increase in porto-caval pressure gradient and allowed a moderate increase in portal flow per unit liver. This effect resulted in protection of the remnant parenchyma without compromising liver regeneration. However, comparing the two groups, only a few parameters reached the statistical significance.

The full potentials of this novel technique could be more evident in a situation where the increase in portal flow per unit mass is higher. Here, the goal was to first test the ring safety in a non-extreme situation to have a better chance to see its effect at day-3 and day-7. The present study demonstrates the high safety profile and the potential efficacy of the MID-AVR ${ }^{\mathrm{TM}}$. Therefore, we started a human clinical trial (phase I/II) registered at (clinicaltrials.gov) under the number (NCT02390713). 


\section{Conclusions:}

The adjustable vascular ring "MID-AVR ${ }^{\mathrm{TM}}$ " applied around the portal vein is a safe, precise, reversible and efficient mean to protect the hepatic micro-architecture during the initial phase of liver regeneration. It seems to delay slightly liver regeneration in a preserved microarchitecture environment, which might result in better hepatic function over the course of regeneration.

Figures legends:

Figure 1: The MID-AVR ${ }^{\mathrm{TM}}$ in its different shapes according to the degree of balloon inflation. (A) the ring is closed while the balloon is completely deflated, (B) the ring is closed while the balloon is inflated with small amount of saline, $(\mathrm{C})$ the ring tends to open while the balloon is inflated with large amount of saline, and (D) The MID-AVRTM is placed around the portal vein and the balloon on the inner surface is inflated with a small amount of saline, the portal vein shows moderate constriction.

Figure 2: Scatter diagram showing animal mortality in both groups stratified according to the change in portal flow per unit liver mass.

Figure 3: (A) porto-caval pressure gradient was significantly higher in the control group compared to the MID-AVRTM group only after hepatectomy $(* * p<0.01)(B)$ the liver weight before and after resection and the regain at day-7 were not significantly different between both groups.

Figure 4: (A) total bilirubin level was higher in the control group than in the MIDAVRTM group, particularly at postoperative day $-5(*=\mathrm{p}<0.05),(\mathrm{B})$ prothrombin 
activity is not significantly different between both groups, (C) the indocyanine green retention test (ICG) at 15 minutes was, after resection or at day-7, similar in the control group and in the MID-AVRTM group.

Figure 5: Biopsies taken from the control (left panel) and ring (right panel) groups (A) H\&E, x200, day-7 after surgery, bridging necrosis, thick cords forming pseudonodules, (B) H\&E, x400, day-7 after surgery, cholangioductal proliferation, (C) Trichrome, x100, day-7, dilatation and hemorrhagic destruction of the sinusoids with surrounding thick parenchymal cords, (D) H\&E, x200, day-7, Normal architecture, (E) H\&E, x400, day-7, normal aspect portal pedicle with mild arterial dilatation, and (F) Trichrome, x100, day-7, conserved architecture with normal thickness cords and portal spaces.

Figure 6: Analysis of hepatocyte proliferation in regenerating pig livers and reconstruction and quantification of bile canalicular network in regenerating pig livers. (A) There are increased numbers of Ki-67 labelled hepatocyte nuclei at day-3 compared to day- 0 , which decreased again at day-7 (Scale bars are $50 \mu \mathrm{m}$ ). Overview images are included in the supplemental figure 1. (B) Quantification of Ki-67 positive hepatocyte nuclei in five fields per specimen. Five to six pigs were used per time point, namely, 0, 3 and 7 days after partial hepatectomy. (C) Examples of reconstructed pig livers. Blue (left column): nuclei, green (middle column): bile canalicular network and merge (right column). The corresponding reconstructions of control, 7 days after partial hepatectomy with MID-AVRTM and 7 days after partial hepatectomy without MID-AVRTM are shown in Supplemental movies 2, 3 and 4, respectively. Scale bars are $50 \mu \mathrm{m}$ and (D) Length of bile canalicular network in a given volume is shown. The bile canaliculi length is not influenced by application of MID-AVRTM ring. ${ }^{*} \mathrm{p}<0.05$ and ${ }^{* *} \mathrm{p}<0.01$. 


\section{References:}

1. Reichen J. The Role of the Sinusoidal Endothelium in Liver Function. News Physiol Sci 1999; 14:117-121.

2. Ding B-S, Nolan DJ, Butler JM, et al. Inductive angiocrine signals from sinusoidal endothelium are required for liver regeneration. Nature 2010; 468:310-315.

3. Niiya T, Murakami M, Aoki T, et al. Immediate increase of portal pressure, reflecting sinusoidal shear stress, induced liver regeneration after partial hepatectomy. J Hepatobiliary Pancreat Surg 1999; 6:275-280.

4. Glanemann M, Eipel C, Nussler AK, et al. Hyperperfusion syndrome in small-for-size livers. Eur Surg Res ; 37:335-341.

5. Asencio JM, Vaquero J, Olmedilla L, et al. "Small-for-flow" syndrome: shifting the “size" paradigm. Med Hypotheses 2013; 80:573-577.

6. Eshkenazy R, Dreznik Y, Lahat E, et al. Small for size liver remnant following resection: prevention and management. Hepatobiliary Surg Nutr 2014; 3:303-312. 
7. Adams RB, Aloia TA, Loyer E, et al. Selection for hepatic resection of colorectal liver metastases: expert consensus statement. HPB (Oxford) 2013; 15:91-103.

8. Vasavada BB, Chen CL, Zakaria M. Portal flow is the main predictor of early graft dysfunction regardless of the GRWR status in living donor liver transplantation - a retrospective analysis of 134 patients. Int J Surg 2014; 12:177-180.

9. Allard M-A, Adam R, Bucur P-O, et al. Posthepatectomy portal vein pressure predicts liver failure and mortality after major liver resection on noncirrhotic liver. Ann Surg $2013 ; 258: 822-829$.

10. Golse N, Bucur PO, Adam R, et al. New paradigms in post-hepatectomy liver failure. J Gastrointest Surg 2013; 17:593-605.

11. Michalopoulos GK. Liver regeneration after partial hepatectomy: critical analysis of mechanistic dilemmas. Am J Pathol 2010; 176:2-13.

12. Kishi Y, Abdalla EK, Chun YS, et al. Three hundred and one consecutive extended right hepatectomies: evaluation of outcome based on systematic liver volumetry. Ann Surg 2009; 250:540-548. 
13. Truant S, Oberlin O, Sergent G, et al. Remnant liver volume to body weight ratio > or $=0.5 \%$ : A new cut-off to estimate postoperative risks after extended resection in noncirrhotic liver. J Am Coll Surg 2007; 204:22-33.

14. Mullin EJ, Metcalfe MS, Maddern GJ. How much liver resection is too much? Am J Surg 2005; 190:87-97.

15. Van Lienden KP, van den Esschert JW, de Graaf W, et al. Portal vein embolization before liver resection: a systematic review. Cardiovasc Intervent Radiol 2013; 36:25-34.

16. Hoekstra LT, van Lienden KP, Doets A, et al. Tumor progression after preoperative portal vein embolization. Ann Surg 2012; 256:812-817.

17. Al-Sharif E, Simoneau E, Hassanain M. Portal vein embolization effect on colorectal cancer liver metastasis progression: Lessons learned. World J Clin Oncol 2015; 6:142146.

18. Troisi R, de Hemptinne B. Clinical relevance of adapting portal vein flow in living donor liver transplantation in adult patients. Liver Transpl 2003; 9:S36-41. 
19. Hou P, Chen C, Tu Y-L, et al. Extracorporeal continuous portal diversion plus temporal plasmapheresis for "small-for-size" syndrome. World J Gastroenterol 2013; 19:5464-5472.

20. Lo C-M, Liu C-L, Fan S-T. Portal hyperperfusion injury as the cause of primary nonfunction in a small-for-size liver graft-successful treatment with splenic artery ligation. Liver Transpl 2003; 9:626-628.

21. Kim J, Kim C-J, Ko I-G, et al. Splenectomy affects the balance between hepatic growth factor and transforming growth factor- $\beta$ and its effect on liver regeneration is dependent on the amount of liver resection in rats. J Korean Surg Soc 2012; 82:238-245.

22. Marubashi S, Sakon M, Nagano H, et al. Effect of portal hemodynamics on liver regeneration studied in a novel portohepatic shunt rat model. Surgery 2004; 136:10281037.

23. Council. NR. Guide for the Care and Use of Laboratory Animals. Eighth. Washington: Natl Academy Pr; 2007.

24. Court FG, Laws PE, Morrison CP, et al. Subtotal hepatectomy: a porcine model for the study of liver regeneration. J Surg Res 2004; 116:181-186. 
25. Hessheimer AJ, Fondevila C, Taurá $\mathrm{P}$, et al. Decompression of the portal bed and twice-baseline portal inflow are necessary for the functional recovery of a "small-forsize" graft. Ann Surg 2011; 253:1201-1210.

26. Demetris AJ, Kelly DM, Eghtesad B, et al. Pathophysiologic observations and histopathologic recognition of the portal hyperperfusion or small-for-size syndrome. Am J Surg Pathol 2006; 30:986-993.

27. Hammad S, Hoehme S, Friebel A, et al. Protocols for staining of bile canalicular and sinusoidal networks of human, mouse and pig livers, three-dimensional reconstruction and quantification of tissue microarchitecture by image processing and analysis. Arch Toxicol 2014; 88:1161-1183.

28. Friebel A, Neitsch J, Johann T, et al. TiQuant: software for tissue analysis, quantification and surface reconstruction. Bioinformatics 2015; 31:3234-3236.

29. Bosch J, Groszmann RJ, Shah VH. Evolution in the understanding of the pathophysiological basis of portal hypertension: How changes in paradigm are leading to successful new treatments. J Hepatol 2015; 62(1 Suppl):S121-130. 
30. Iguchi K, Hatano E, Yamanaka K, et al. Hepatoprotective effect by pretreatment with olprinone in a swine partial hepatectomy model. Liver Transpl 2014; 20:838-849.

31. Rahbari NN, Garden OJ, Padbury R, et al. Post-hepatectomy haemorrhage: a definition and grading by the International Study Group of Liver Surgery (ISGLS). HPB (Oxford) 2011; 13:528-535.

32. Lautt WW. Regulatory processes interacting to maintain hepatic blood flow constancy: Vascular compliance, hepatic arterial buffer response, hepatorenal reflex, liver regeneration, escape from vasoconstriction. Hepatol Res 2007; 37:891-903.

33. Kjekshus H, Risoe C, Scholz T, et al. Methods for assessing hepatic distending pressure and changes in hepatic capacitance in pigs. Am J Physiol Heart Circ Physiol 2000; 279:H1796-803.

34. Mueller L, Broering DC, Meyer J, et al. The induction of the immediate-early-genes Egr-1, PAI-1 and PRL-1 during liver regeneration in surgical models is related to increased portal flow. J Hepatol 2002; 37:606-612.

35. Nobuoka T, Mizuguchi T, Oshima H, et al. Portal blood flow regulates volume recovery of the rat liver after partial hepatectomy: molecular evaluation. Eur Surg Res 
$2006 ; 38: 522-532$.

36. Pan N, Lv X, Liang R, et al. Suppression of graft regeneration, not ischemia/reperfusion injury, is the primary cause of small-for-size syndrome after partial liver transplantation in mice. PLoS One 2014; 9:e93636.

37. Vasavada B, Chen CL, Zakaria M. Using low graft/recipient's body weight ratio graft with portal flow modulation an effective way to prevent small-for-size syndrome in living-donor liver transplant: a retrospective analysis. Exp Clin Transplant 2014; 12:43742.

38. Umeda Y, Yagi T, Sadamori H, et al. Effects of prophylactic splenic artery modulation on portal overperfusion and liver regeneration in small-for-size graft. Transplantation 2008; 86:673-80.

39. Taniguchi M, Shimamura T, Suzuki T, et al. Transient portacaval shunt for a smallfor-size graft in living donor liver transplantation. Liver Transpl 2007; 13:932-4.

40. Wang X-Q, Xu Y-F, Tan J-W, et al. Portal inflow preservation during portal diversion in small-for-size syndrome. World J Gastroenterol 2014; 20:1021-9. 
41. Troisi R, Hesse UJ, Decruyenaere J, et al. Functional, life-threatening disorders and splenectomy following liver transplantation. Clin Transplant 1999; 13:380-8.

Table 1: Different parameters measured after liver resection and on day-7 post-operative

\begin{tabular}{|c|c|c|c|}
\hline & $\begin{array}{c}\text { MID-AVRTM Group } \\
(\mathrm{n}=8)\end{array}$ & $\begin{array}{c}\text { Control Group } \\
(\mathrm{n}=9)\end{array}$ & $p$ \\
\hline Portal flow (ml/min) & $565 \pm 164$ & $544 \pm 122$ & ns \\
\hline $\begin{array}{l}\text { Portal flow for remnant liver } \\
\mathrm{mass}(\mathrm{ml} / \mathrm{min} / \mathrm{g} * 100 \mathrm{~g})^{\mathrm{a}}\end{array}$ & $372 \pm 97$ & $359 \pm 95$ & ns \\
\hline Arterial flow $(\mathrm{ml} / \mathrm{min})$ & $92 \pm 49$ & $64 \pm 60$ & $\mathrm{~ns}$ \\
\hline Cardiac output (1/min) & $2.6 \pm 0.8$ & $2.3 \pm 0.9$ & ns \\
\hline $\begin{array}{l}\text { Central venous pressure } \\
(\mathrm{mmHg})\end{array}$ & $5.9 \pm 2.0$ & $4.9 \pm 1.6$ & ns \\
\hline Portal pressure $(\mathrm{mmHg})$ & $7.5 \pm 2.4$ & $9.3 \pm 2.3$ & ns \\
\hline Porto-caval gradient (mmHg) & $1.63 \pm 1.3$ & $4.4 \pm 1.5$ & 0.001 \\
\hline ASAT (UI /ml) & $169 \pm 60$ & $197 \pm 110$ & ns \\
\hline ALAT (UI/ml) & $37.3 \pm 8.1$ & $42 \pm 6$ & ns \\
\hline Platelets (X 103/ $\mu 1)$ & $348 \pm 101$ & $312 \pm 31$ & $\mathrm{~ns}$ \\
\hline \multicolumn{4}{|c|}{ Analysed parameters at animal sacrifice } \\
\hline Animal weight $(\mathrm{kg})$ & $30 \pm 3.8$ & $30 \pm 6.0$ & $\mathrm{~ns}$ \\
\hline Liver weight $(\mathrm{g})^{\mathrm{b}}$ & $507 \pm 77$ & $478 \pm 37$ & ns \\
\hline Portal flow (ml/min) & $710 \pm 224$ & $698 \pm 190$ & ns \\
\hline $\begin{array}{l}\text { Portal flow per unit liver mass } \\
(\mathrm{ml} / \mathrm{min} / \mathrm{g} * 100 \mathrm{~g})\end{array}$ & $149 \pm 54$ & $93 \pm 79$ & ns \\
\hline Hepatic arterial flow (ml/min) & $151 \pm 61$ & $93 \pm 79$ & ns \\
\hline Cardiac output (1/min) & $2.7 \pm 1.2$ & $2.8 \pm 1.3$ & ns \\
\hline $\begin{array}{l}\text { Central venous pressure } \\
(\mathrm{mmHg})\end{array}$ & $4.7 \pm 1.7$ & $4.8 \pm 1.1$ & ns \\
\hline Portal pressure $(\mathrm{mmHg})$ & $8.2 \pm 1.3$ & $9.4 \pm 0.9$ & ns \\
\hline Porto-caval gradient $(\mathrm{mmHg})$ & $3.5 \pm 1$ & $4.6 \pm 1.7$ & ns \\
\hline$\% 15$ min ICG retention ${ }^{c}$ & $28 \pm 18$ & $38 \pm 7$ & ns \\
\hline ASAT (UI /ml) & $83 \pm 61$ & $67 \pm 50$ & ns \\
\hline $\operatorname{ALAT}(\mathrm{UI} / \mathrm{ml})$ & $72 \pm 30$ & $66 \pm 17$ & ns \\
\hline Platelets $\left(X 10^{3} / \mu \mathrm{l}\right)$ & $375 \pm 30$ & $435 \pm 133$ & $\mathrm{~ns}$ \\
\hline
\end{tabular}

a The weight of the remnant liver was estimated based on the fact that resection of the left and median lobes is nearly equal to $75 \%$.

$\mathrm{b}$ Whole liver weight is the sum of the resected liver weight and the calculated liver weight.

c ICG= Indocyanine green 
Figures:

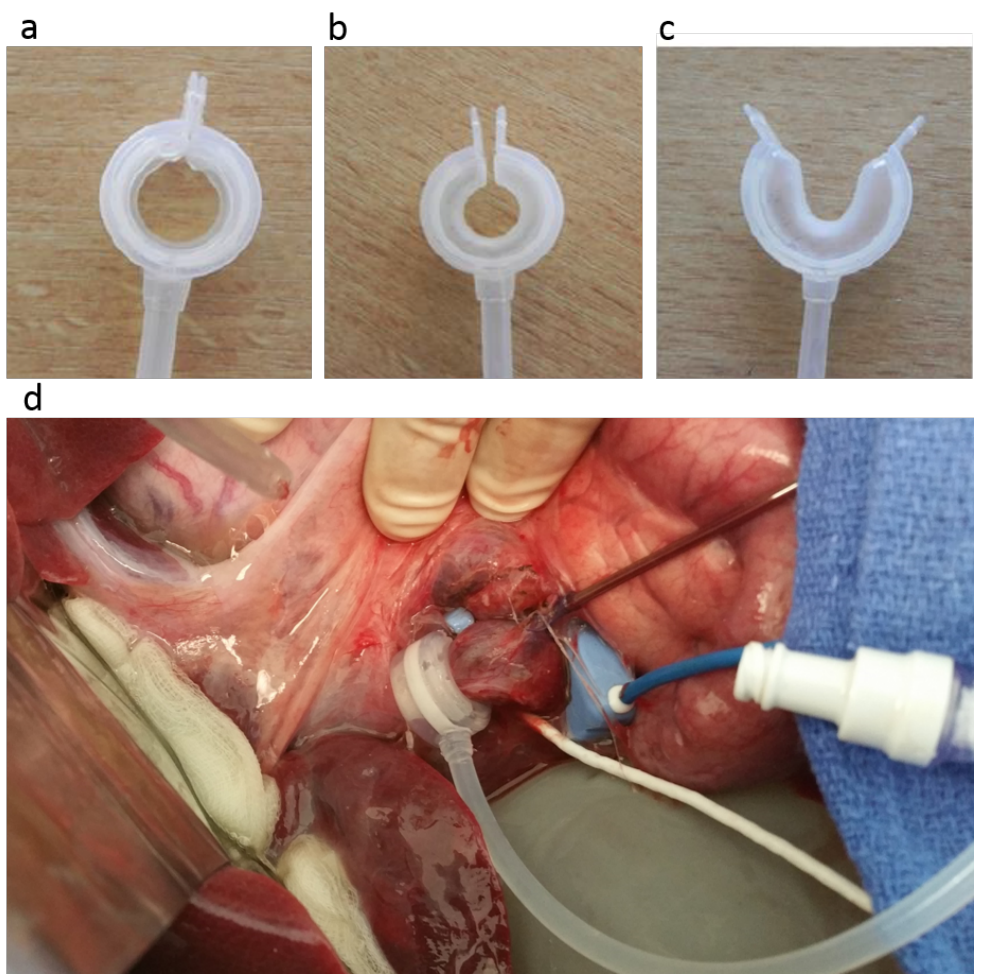

Figure 1: The MID-AVR in its different shapes according to the degree of balloon inflation. A, The ring is closed while the balloon is completely deflated. B, The ring is closed while the balloon is inflated with small amount of saline. $\mathrm{C}$, The ring tends to open while the balloon is inflated with large amount of saline. D, The MID- AVR is placed around the portal vein and the balloon on the inner surface is inflated with a small amount of saline, the portal vein shows moderate constriction. 


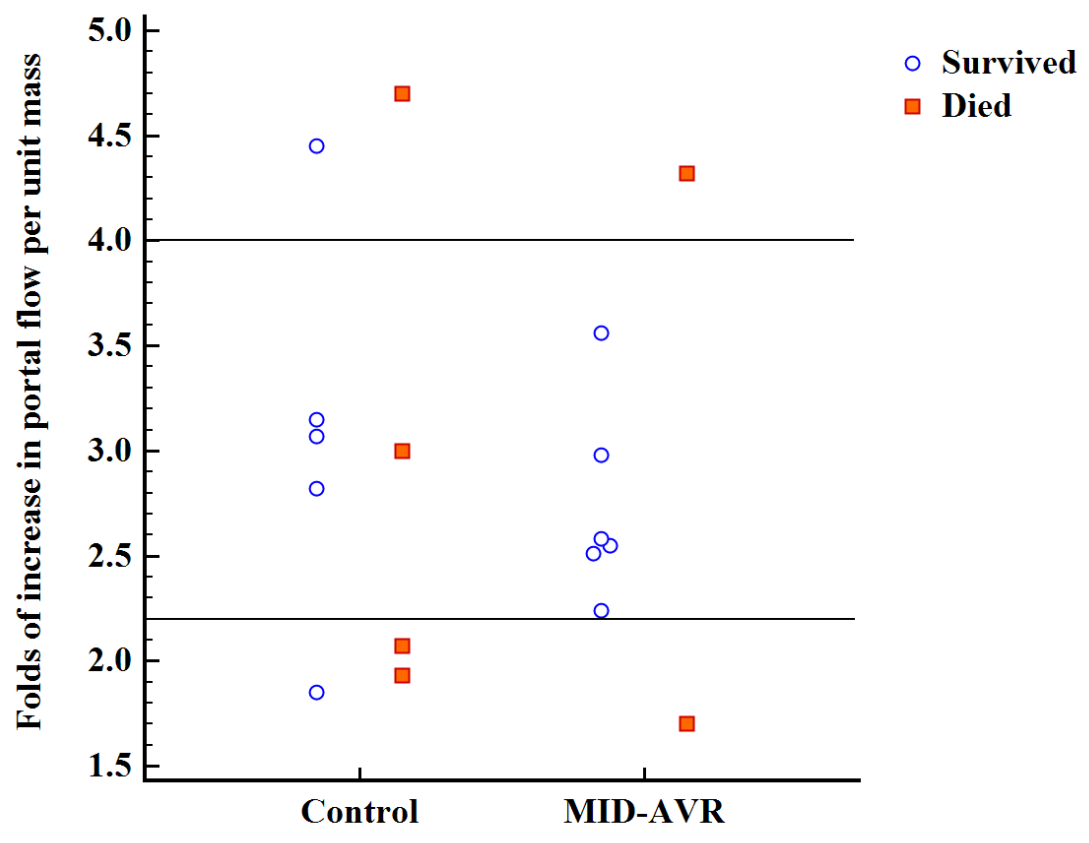

Figure 2: Scatter diagram showing animal mortality in both groups stratified according to the change in portal flow per unit liver mass. 


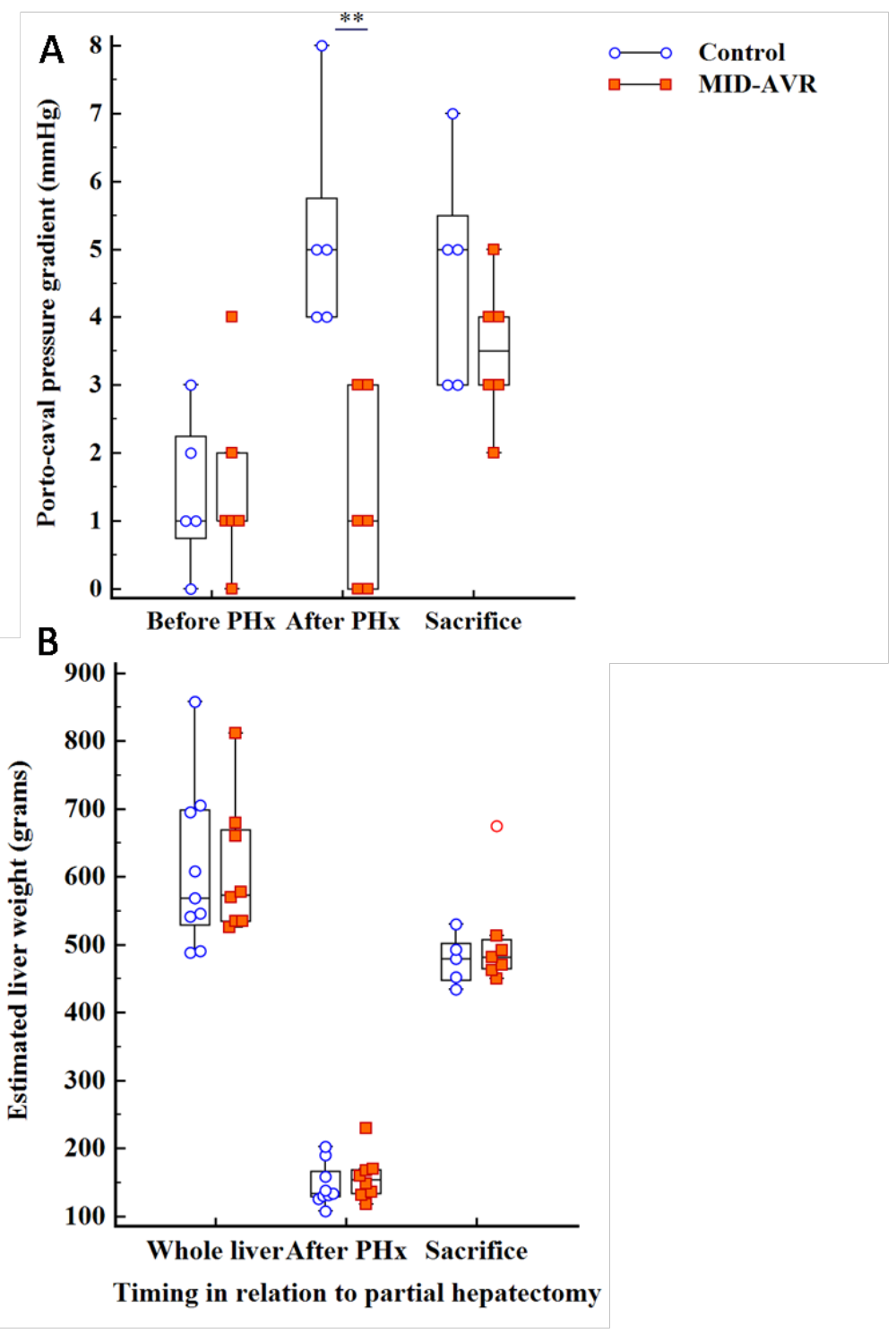

Figure 3: A, Portocaval pressure gradient was significantly higher in the control group compared to the MID-AVR group only after hepatectomy $\left({ }^{* *} \mathrm{P}<0.01\right)$. B, The liver weight before and after resection and the regain on day 7 were not signifi- cantly different between both groups. 

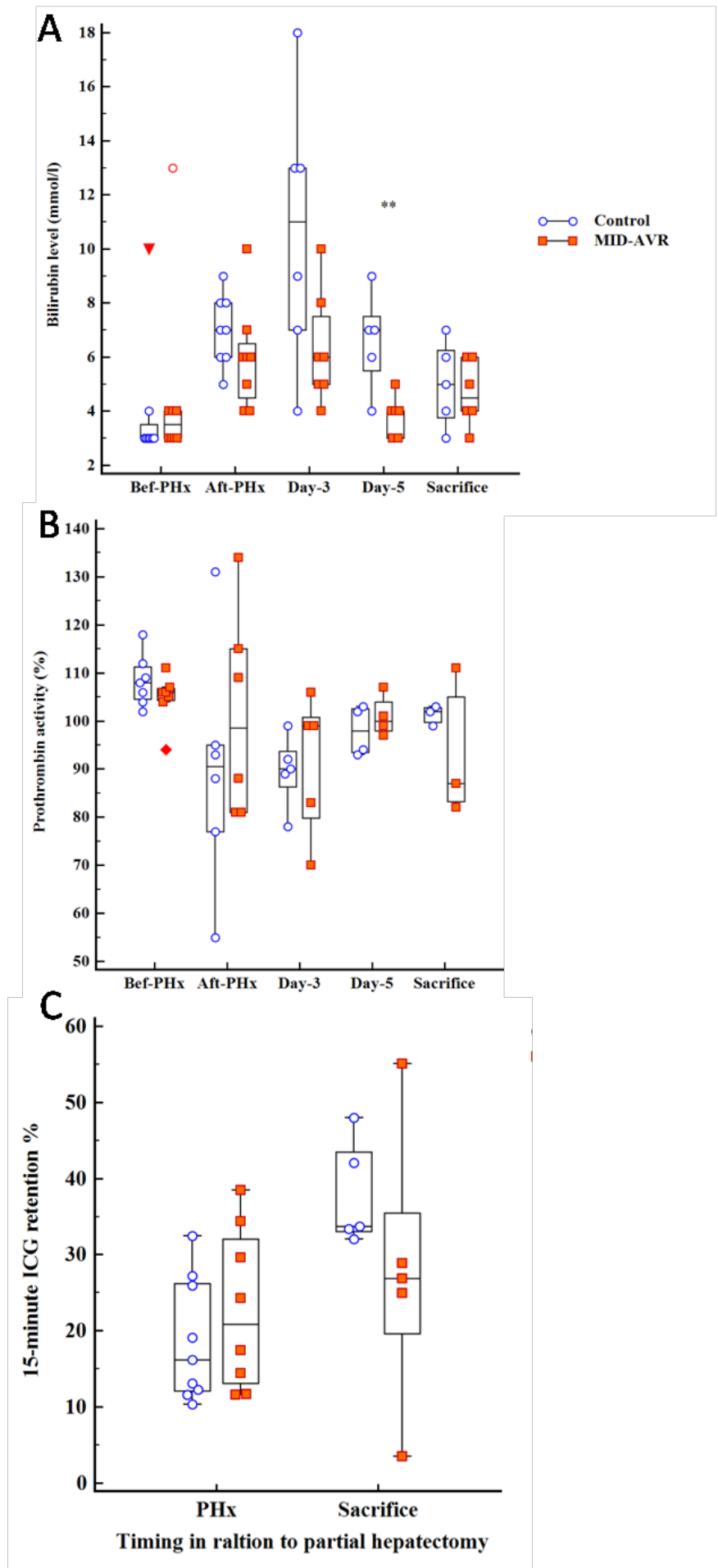

Figure 4: A, Total bilirubin level was higher in the control group than in the MID-AVR group, particularly on postoperative day $5(\mathrm{P}<0.05)$. $\mathrm{B}$, Prothrombin activity is not significantly different between both groups. $\mathrm{C}$, The indocyanine green retention test (ICG) at 15 minutes was, after resection or on day 7 , similar in the control group and in the MID-AVR group. 


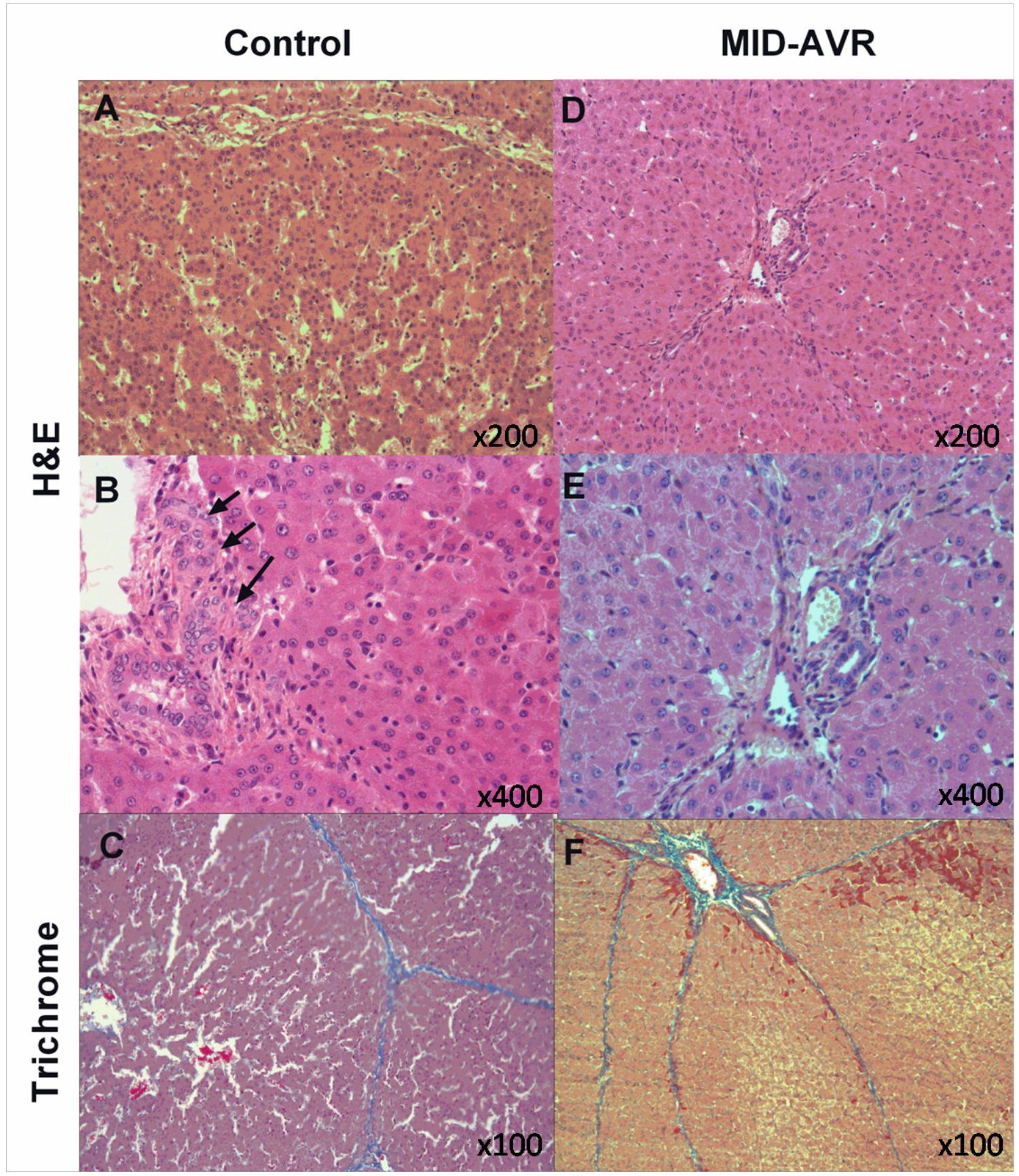

Figure 5: Biopsies taken from the con- trol (left panel) and ring (right panel) groups: A, $\mathrm{H} \& \mathrm{E}$, x200, day 7 after surgery, bridging necrosis, thick cords forming pseudonodules. B, H\&E, x400, day 7 after surgery, cholangioductal pro- liferation. C, Trichrome, x100, day 7, dilatation and hemorrhagic destruction of the sinusoids with surrounding thick parenchymal cords. D, H\&E, x200, day 7, normal architecture. E, H\&E, x400, day 7, normal aspect portal pedicle with mild arterial dilatation. F, Trichrome, x100, day 7 , conserved architecture with normal thickness cords and portal spaces. 


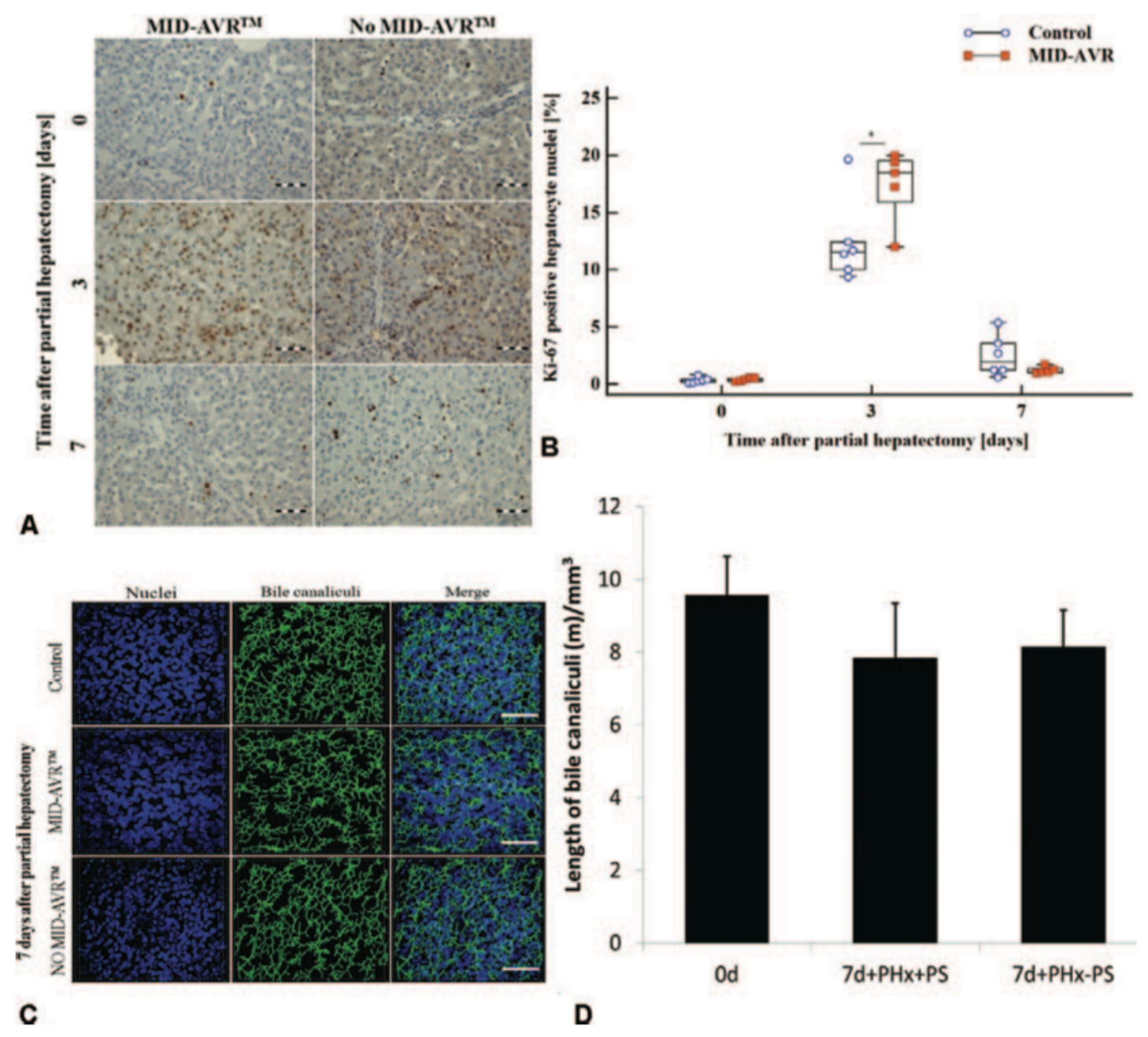

Figure 6: Analysis of hepatocyte pro- liferation in regenerating pig livers and reconstruction and quantification of bile canalicular network in regenerating pig livers. A, There are increased numbers of Ki-67 labeled hepatocyte nuclei on day 3 compared to day 0 , which decreased again on day 7 (scale bars are $50 \mathrm{~mm}$ ). Overview images are included in the supplemental Figure 1, http://links. lww.com/SLA/B173. B, Quantification of Ki-67 positive hepatocyte nuclei in 5 fields per specimen. Five to six pigs were used per time point, namely, 0,3 , and 7 days after partial hepatectomy. C, Examples of reconstructed pig livers. Blue (left column): nuclei, green (middle column): bile canalicular network and merge (right column). The correspond- ing reconstructions of control, 7 days after partial hepatectomy with MID- AVR and 7 days after partial hepatectomy without MID-AVR are shown in Supple- mental movies 2, 3, and 4, http://links. lww.com/SLA/B175, http://links.lww. com/SLA/B176, http://links.lww.com/ SLA/ B177, respectively. Scale bars are $50 \mathrm{~mm}$. D, Length of bile canalicular net- work in a given volume is shown. The bile canaliculi length is not influenced by application of MID-AVR ring. ${ }^{*} \mathrm{P}<0.05$ and ${ }^{* *} \mathrm{P}<0.01$. 